\title{
MODELING AND PRESSURE PREDICTION OF A BLOCK OF THE JEQUITINHONHA BASIN
}

\author{
Wildney W.S. Vieira, Lourenildo W.B. Leite and Boris Sibiryakov
}

\begin{abstract}
We present in this paper a seismic-stratigraphical framework for pressure prediction for oil and gas exploration in sedimentary basins, that is based on seismic information, with application to a marine part of the Jequitinhonha basin (east of the State of Bahia, Brazil). For this purpose it is necessary the knowledge of velocity distributions (compressional $v_{P}$ and shear $v_{S}$ ), that can be obtained from seismic sections, petrophysical information and empirical models, and the distribution of density $(\rho)$. We show some details of the theoretical modeling method, with an example that shows how pressure varies in the subsurface. We highlight that pressure that does not necessarily increase linearly with depth, but in a complex way that requires specific numerical calculations to be able to see important details related to a reservoir and surroundings. The model considers the vertical gravity load as the source of pressure in the geological formations, and in the present case we do not take into account the effects of formation curvatures, faulting, diagenesis and lateral tectonic events. We show that the meaning of cap rock of a reservoir is not to be related to lithology, but directly to the physics of high and low pressure zones that results from the mechanics of solid bodies under a stress-strain system; in this direction, and as a curiosity, we also show how a basement rock can become a reservoir. It is important to emphasize that an accurate prediction needs a 3D model for a meaningful and complete practical application, in the other hand, it requires handling large matrices.
\end{abstract}

Keywords: basin modeling, seismic velocity analysis, pressure prediction, empirical models.

RESUMO. Este artigo tem como objetivo compor uma estratégia sismo-estratigráfica onde se visa a predição de pressão na exploração de gás e óleo em bacias sedimentares com base em informação sísmica, com aplicação a uma parte marinha da bacia de Jequitinhonha (leste do estado da Bahia, Brasil). Para esta finalidade é necessário o conhecimento das distribuições de velocidades (compressiva $v_{P}$ e cisalhante $v_{S}$ ) que podem ser obtidas a partir de seções sísmicas, informações petrofísicas e modelos empíricos, e da distribuição de densidade $(\rho)$. Apresentamos alguns detalhes da modelagem teórica, e um exemplo para mostrar como a pressão varia na subsuperfície, onde destacamos que a predição de pressão não aumenta necessariamente linearmente, mas de uma forma complexa que requer cálculos numéricos específicos para poder obter detalhes relacionados a um reservatório. 0 modelo apresenta a carga vertical da gravidade como a fonte de pressão nas formações geológicas, e neste caso não se leva em consideração os efeitos de curvaturas das camadas, falhas, diagênese e eventos tectônicos laterais. Mostramos que o significado de uma rocha selante de um reservatório não deve estar relacionado à litologia, mas simplesmente à física de zonas de alta e baixa pressão como resultado da mecânica dos corpos sólidos sob o sistema de tensão-deformação; neste sentido, e como curiosidade, mostramos também como uma rocha do embasamento pode se tornar um reservatório de óleo e gás. É importante observar que uma predição com precisão necessita de um modelo 3D para uma aplicação prática significativa e completa, mas que requer o manuseio de matrizes grandes.

Palavras-chave: modelagem de bacia, análise de velocidade sísmica, predição de pressão, modelos empíricos.

Universidade Federal do Pará, Campus Universitário do Guamá, Instituto de Geociências, 66075-110 Belém, PA, Brazil. Phone: +55(91) 3201-7693; Fax: +55(91) 3201-7609 - E-mails: wildneyvieira@gmail.com; Iwbleite@gmail.com; SibiryakovBP@ipgg.nsc.ru 


\section{INTRODUCTION}

This paper presents a numerical experiment of basin modeling and pressure prediction, where we use four seismic lines of the Jequitinhonha basin located to the east of the State of Bahia (Brazil), marine side. The lines used were separated into two groups: three lines in the NE-SW (L214-266, L214-268, L214270), and one line in the NW-SE direction (L214-297), in order to construct what we call an empirical 3D pressure model. This means, since the surveyed lines are independent, in principle we cannot construct a single subsurface distribution of velocities $\left(v_{P}\right.$ and $\left.v_{S}\right)$ and density $(\rho)$. Therefore, we interpolated and extrapolated the results obtained for the individual time sections to construct a limited block, but that can still give plausible and consistent geological information. A precursor to the present paper describing the basic and fundamental theory can be followed in Leite et al. (2016).

The methodology was composed of velocity analysis, CRS stacking and migration (Mann, 2002), geometrical interpretation of reflectors, and the construction of a controlled empirical model for $v_{P}, v_{S}$, and density $\rho$, leading to the prediction of pressure in the subsurface. The goal is the mapping of areas of low (example, rock reservoir) and high (example, rock generator) pressure zones that act as natural pumps for the accumulation of fluids (Payton, 1977). In this direction, Sibiryakov et al. (2014) have also presented the basic theory, and Silva (2016) presented results that also serves as precursors to the present work. Pressure prediction, in sedimentary basin modeling for oil and gas exploration, is a very attractive subject that can contribute to complex geological understanding, and to participate in the location of a borehole for a successful productive well.

The interpretation of the seismic sections was to recognize patterns existing between the main reflective events, where four basic principles were considered: resolution (vertical), continuity (horizontal), texture (of the intervals between dominant events), and frequency content (low, medium, high).

The so-called Regência Petroleum System is responsible for all hydrocarbon occurrences in the Jequitinhonha basin (Mello et al., 1994). However, we should mention that it is necessary to emphasize that a rock is a reservoir candidate if it fulfills the mechanical-static conditions of pressure state, therefore we may put some literature in contradictory positions in some details about the reservoir stratigraphy.

Basin modeling aiming at oil and gas exploration contains many theoretical aspects related to engineering, geology, geochemistry and geophysics, in order to characterize a reservoir as described by Hantschel \& Kauerauf (2009). Ameem (2003) presents a special bibliography for methods and theories on crustal stress studies, where basins are included. But, it should be clear here that we follow the development of a specific data driven method that is based on $v_{\mathrm{P}}(\mathbf{x}), v_{\mathrm{S}}(\mathbf{x})$ and $\rho(\mathbf{x})$ as a priori knowledge, in order to map low pressure zones that are important to locate a successful drilling zone for oil and gas exploration.

\section{METHODOLOGY}

We start describing part of the data information contained in Figure 1, where we show the interpreted sections of the CRS zero offset stack (normal incidence sections). The geometrical interpretation was based on reflection events with clear lateral continuity, and on the texture of the macro intervals, where the sequences and structures are indicated by letters and numbers. The interpretation outlines basement highs, the layered sequence displaying lateral variations and specific textures. The flat top of the figures shows clearly the contact water/sediments with similar behavior for a flatter platform followed by the main marine continental slope. We point out here that the three parallel lines (66, 68 and 70 ) were used to construct the empirical 3D cube, while the transversal line, 97 , was only used to help the interpretation process.

\section{Pressure Prediction}

The main physical parameter governing pressure prediction is given by the measure of discontinuity represented by the velocity ratio $\gamma=v_{S} / v_{P}$ along the interfaces. Sibiryakov et al. (2015) deals with different aspects of this special method, where is described that, as a result of the $\gamma$ ratio behavior, an anticline is not necessarily the only structural condition for potentiating a zone to be an oil and gas accumulator (reservoir).

The continuous linear elastic stress, $\sigma=\sigma(x, y, z)$, and deformation, $\epsilon=\epsilon(x, y, z)$, fields described as tensors functions of space, and represented by nine components. For the general anisotropic media, the tensors $\sigma$ and $\epsilon$ obey the spatial coordinate rotation by the relation: $\sigma_{i j}=\sum_{k, l} a_{j k l} \sigma_{k l}^{\prime}$, and $\epsilon_{i j}=\sum_{k, l} b_{j j k l} \epsilon_{k l}^{\prime}$, where the coefficients $a_{i j k l}$ and $b_{i j k l}$ define the new plane with respect to a reference system. The linear elastic relationship between stress and strain is given by the generalized Hooke's law: $\sigma_{i j}=\sum_{k} c_{i j k l} \epsilon_{k l}$. In this description, the first index $(i)$ in $\sigma_{i j}$ and $\epsilon_{j j}$ represents the plane direction, and the second $(j)$ the component direction. The stress state is represented at a point $Q$ by a matrix $\mathbf{S}$ with elements $\sigma_{i j}$.

Now we describe internal aspects of the stress fields followed by the constituent relations for pressure prediction in layered solid subsurface. 


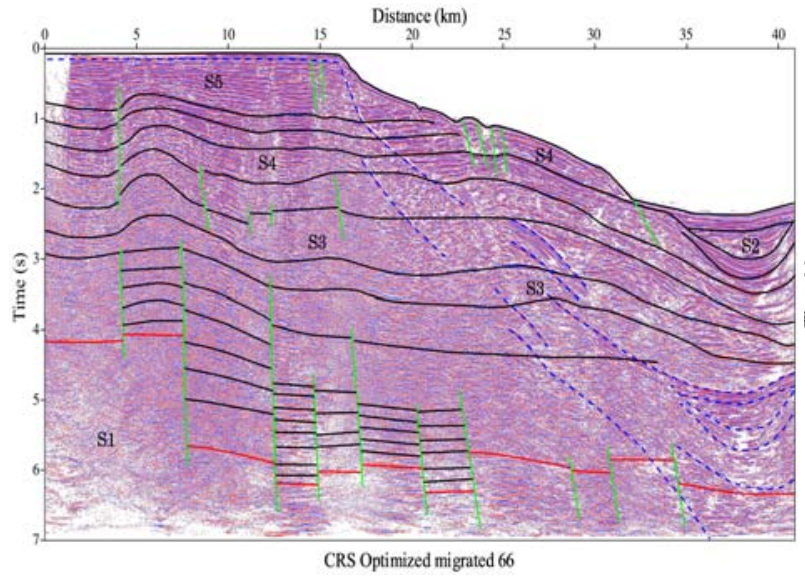

(a) Line 66 .

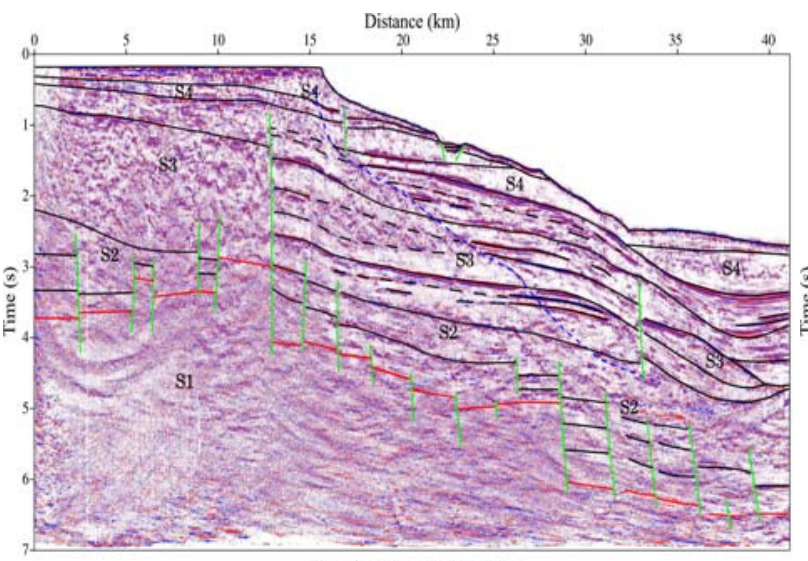

CRS Optimized migrated 70

(c) Line 70.

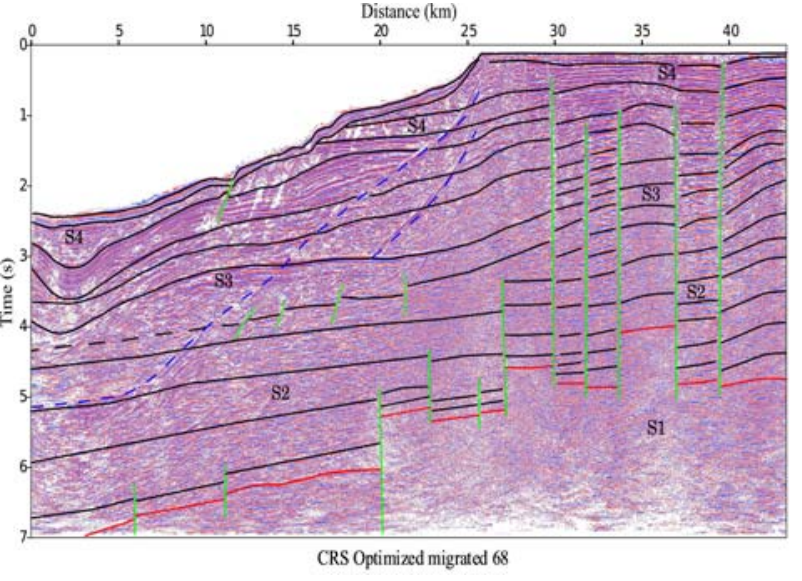

(b) Line 68

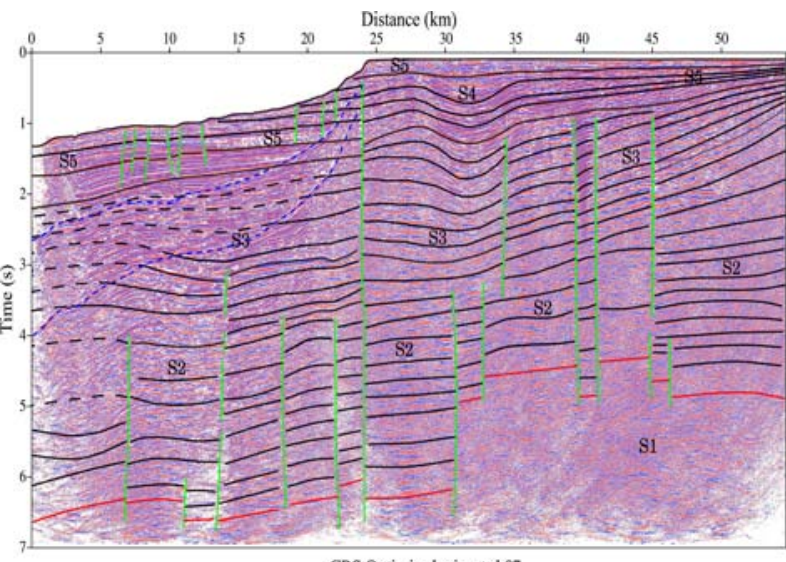

CRS Optimized migrated 97

(d) Line 97.

Figure 1 - Seismic interpretation of the used sections.

\section{Stress states}

The stress matrix can be decomposed in three parts: $\mathbf{S}=\mathbf{S}_{\mathbf{0}}+\mathbf{S}_{\mathbf{D}} \mathbf{S}_{\mathbf{N}}$, which allow a physical interpretation (Persen, 1975). For the state $\mathbf{S}_{\mathbf{0}}$ we simply have that $\mathbf{S}_{\mathbf{0}}=\left\{P_{H} \delta_{i j}\right\}$, where $P_{H}=\frac{1}{3}\left(\sigma_{x x}+\sigma_{y y}+\sigma_{z z}\right)$ is the sum of the normal stresses which defines the so-called hydrostatic pressure, and this state is present in any plane around the point $Q$. For the state $\mathbf{S}_{\mathbf{D}}$ we have that:

$$
\mathbf{S}_{\mathbf{D}}=\left[\begin{array}{ccc}
\sigma_{x x}-P_{H} & \frac{1}{2}\left(\sigma_{x y}+\sigma_{y x}\right) & \frac{1}{2}\left(\sigma_{x z}+\sigma_{z x}\right) \\
\frac{1}{2}\left(\sigma_{x y}+\sigma_{y x}\right) & \sigma_{y y}-P_{H} & \frac{1}{2}\left(\sigma_{y z}+\sigma_{z y}\right) \\
\frac{1}{2}\left(\sigma_{x z}+\sigma_{z x}\right) & \frac{1}{2}\left(\sigma_{z y}+\sigma_{y z}\right) & \sigma_{z z}-P_{H}
\end{array}\right] .
$$

Applying the symmetry property to the above matrix (1): $\sigma_{x y}=\sigma_{y x}, \sigma_{x z}=\sigma_{z x}, \sigma_{y z}=\sigma_{z y}$, $\mathbf{S}_{\mathbf{D}}$, results in a null state, i.e., $\mathbf{S}_{\mathbf{D}}=\mathbf{0}$. For the state $\mathbf{S}_{\mathbf{N}}$ we have that:

$$
\mathbf{S}_{\mathbf{N}}=\left[\begin{array}{ccc}
0 & \frac{1}{2}\left(\sigma_{x y}-\sigma_{y x}\right) & \frac{1}{2}\left(\sigma_{x z}-\sigma_{z x}\right) \\
\frac{1}{2}\left(\sigma_{x y}-\sigma_{y x}\right) & 0 & \frac{1}{2}\left(\sigma_{y z}-\sigma_{z y}\right) \\
\frac{1}{2}\left(\sigma_{x z}-\sigma_{z x}\right) & \frac{1}{2}\left(\sigma_{z y}-\sigma_{y z}\right) & 0
\end{array}\right] .
$$


By the same principle, applying the symmetry property, the state $\mathbf{S}_{\mathbf{N}}$ simplifies to:

$$
\mathbf{S}_{\mathbf{N}}=\left[\begin{array}{ccc}
\sigma_{x x}-P_{H} & \sigma_{x y} & \sigma_{x z} \\
\sigma_{y x} & \sigma_{y y}-P_{H} & \sigma_{x z} \\
\sigma_{z x} & \sigma_{z x} & \sigma_{z z}-P_{H}
\end{array}\right]
$$

what places the deviatoric state along the diagonal (normal stresses), from where the hydrostatic state is subtracted, remaining the non-hydrostatic state.

For an isotropic, linear elastic, medium, the relation between stress and strain is represented by Hooke's law in the special form: $\sigma_{i j}=\lambda \theta \delta_{i j}+2 \mu \epsilon_{i j}$, where $\lambda$ and $\mu$ are the Lamé's elastic parameters, and $\delta_{i j}$ the Kronecker delta $\left(\delta_{i j}=0\right.$, if $i \neq j$ and $\delta_{i j}=1$, if $i=j$ ). The non-dimensional parameter $\theta$ represents the cubic dilation, and is given by the divergence of the displacement vector $\mathbf{u}$ as:

$$
\theta=\nabla \cdot \mathbf{u}=\frac{\partial u_{x}}{\partial x}+\frac{\partial u_{y}}{\partial y}+\frac{\partial u_{z}}{\partial z} .
$$

The non-dimensional tensor components $\epsilon_{i j}$ are defined in terms of the displacement components $u_{i}$ in the form:

$$
\epsilon_{i j}=\frac{1}{2}\left(\frac{\partial u_{i}}{\partial x_{j}}+\frac{\partial u_{j}}{\partial x_{i}}\right) .
$$

Besides, the linear shear-extensional tensor:

$$
\varphi_{i j}=\frac{1}{2}\left(\frac{\partial u_{i}}{\partial x_{j}}-\frac{\partial u_{j}}{\partial x_{i}}\right),
$$

is a non-dimensional rotation tensor.

We may notice that, once the displacement field is known, the stress and deformation tensors can be calculated by applying spatial derivatives.

\section{Governing equations}

The differential equations that represent static stress state of a physical particle of the subsurface, and to be integrated, is given by a system of related forces as:

$$
\left\{\begin{array}{l}
\frac{\partial \sigma_{x x}}{\partial x}+\frac{\partial \sigma_{x y}}{\partial y}+\frac{\partial \sigma_{x z}}{\partial z}=p_{x}(x, y, z), \\
\frac{\partial \sigma_{y x}}{\partial x}+\frac{\partial \sigma_{y y}}{\partial y}+\frac{\partial \sigma_{y z}}{\partial z}=p_{y}(x, y, z), \\
\frac{\partial \sigma_{z x}}{\partial x}+\frac{\partial \sigma_{z y}}{\partial y}+\frac{\partial \sigma_{z z}}{\partial z}=p_{z}(x, y, z),
\end{array}\right.
$$

resumed to the form,

$$
\begin{gathered}
\frac{\partial \sigma_{i j}}{\partial x_{j}}=p_{j}(x, y, z), \\
(i, j=1,2,3) \quad \text { or } \quad(i, j=x, y, z),
\end{gathered}
$$

where $p_{j}(x, y, z)$ can represent a tectonic (external) horizontal $(x$ and $y$ ) stress-pressure field on the block, but the horizontal components are usually defined as null, and the vertical component is defined as the gravity loading, $p_{z}=\rho(z) g(z)$, that can be further simplified to $p_{z}=\rho g$, with $\rho g$ constant for the considered thickness column. In other words, for the present approach the vertical gravity load is considered the only one responsible for producing the basin stress field, therefore the horizontal tectonic components are null, $p_{x}(x, y, z)=0$ and $p_{y}(x, y, z)=0$, and they could be introduced if there is a difference between calculated and real horizontal stresses.

The present model is related to the wave propagation in a perfect linear elastic medium, where the elasto-dynamic equations of motion have in the right hand side the acceleration term, and a ceased source term; to recall the case, the system of equations of dynamic state is resumed to the form:

$$
\frac{\partial \sigma_{i j}}{\partial x_{j}}=\rho \frac{\partial^{2} u_{i}}{\partial t^{2}} .
$$

This means that the temporal-spatial stress variation is related to the inertial forces (per unit volume), not considering internal forces in this case. It should be clear that we are not studying wave propagation in pre-stressed media, but the case of static deformation, that could be analyzed based on the dynamic equation of motion for the limit of zero frequency (static condition).

The basic seismic velocities ( $\mathrm{P}$ and $\mathrm{S}$ ) for elastic, homogeneous, isotropic media are given by:

$$
v_{\mathrm{P}}=\sqrt{(\lambda+2 \mu) / \rho}, \quad v_{\mathrm{S}}=\sqrt{\mu / \rho},
$$

where $\mu$ is the shear module, $\rho$ is the volumetric density, and $\lambda$ is related to the bulk and shear modules. From the above relations, the modules can calculated by:

$$
\mu=v_{\mathrm{S}}^{2} \rho, \quad \lambda=v_{\mathrm{P}}^{2} \rho-2 \mu=\left(v_{\mathrm{P}}^{2}-2 v_{\mathrm{S}}^{2}\right) \rho .
$$

The ratio $\gamma=v_{\mathrm{S}} / v_{\mathrm{P}}=\frac{\mu}{\lambda+2 \mu}$ does not depend on $\rho$, and represent a sampling over the $\mu$ and $\lambda$ parameters. The waves sample the elastic parameters in the subsurface, and the parameters are considered to vary only spatially, and not with the propagation time.

Density is usually admitted as a parameter that varies slowly with depth, from the Earth's surface to the top of the target interface; but, in some geological situations the density discontinuity, across an interface, can be relatively high. In the present case of vertical sections, the density is modeled in $2 \mathrm{D}, \rho=\rho(x, z)$, and is integrated in the calculation grid.

The used system of differential equations to be integrated corresponds to the description of the static problem [no time variation 
(4)]. These equations govern the mechanics of solids under the influence of gravity (may include tectonic forces over the selected block), and they are summarized to the form: $\frac{\partial \sigma_{i j}}{\partial x_{j}}=\rho g \delta_{3 j}$. This means that the sum of the horizontal stress variations are zero, and the vertical component is controlled by the gravity loading in the subsurface expressed in terms of force per unit area, and set to $p_{z}=\rho g$. That is, the lateral tectonic stresses are not taken into account in this model, besides we do not have such information for the area. Also, in other macro cases the quantities $\rho$ and $g$ can be considered as spatial functions; that is, $\rho=\rho(x, y, z)$ and $g=g(x, y, z)$.

We describe first a model for the geological medium as represented by plane-horizontal layers along the vertical $z$ axis at each point of the $x$ axis of the profile. The equilibrium equation for the linear elastic medium for each layer is $\frac{\partial \sigma_{i k}}{\partial x_{k}}=\rho g_{i}$, where $\sigma_{i k}$ represents the components of the stress tensor, $\rho$ the rock density, and $g_{i}$ the gravity acceleration. For the case of vertical gravity, $g_{i=z}(z) \approx g$, admitted as constant for a small depth variation, the equilibrium equation is simplified to the form $\frac{\partial \sigma_{z z}}{\partial x_{z}}=\rho g$. This equation has an elementary solution that is given by:

$$
\left.\sigma_{z z}\right|_{z=z_{0}}=P_{z}=\int_{z=0}^{z=z_{0}} \rho g d z=\rho g z_{0}=P_{0}\left(z_{0}\right),
$$

where $P_{0}=\rho g z_{0}$ is the overload rock weight per unit area; i.e., the vertical pressure due to the overload at any depth $z_{0}$.

For the present model, where the vertical stress, $\sigma_{z z}(z)$, is defined as only due to the overloading layer; i.e., $\sigma_{z z}=$ $P_{z}=P_{0}(z)$. The horizontal stress, $\sigma_{x x}(z)$, considering that $\sigma_{y y}=\sigma_{x x}$, is smaller than the vertical stress, $\sigma_{z z}$, and we can demonstrate it to be given by:

$$
\sigma_{x x}=P_{x}=P_{0}\left(1-2 \gamma^{2}\right),
$$

where $P_{0}=P_{0}(z), \gamma=\gamma(z)=v_{\mathrm{S}}(z) / v_{\mathrm{P}}(z)$.

The scalar invariant hydrostatic pressure field, $P(z)=P_{H}$, was defined in the matrix (1) as the average:

$$
P=P_{H}=\frac{1}{3}\left(\sigma_{x x}+\sigma_{y y}+\sigma_{z z}\right) .
$$

Using Hooke's generalized law, it is demonstrated that $P=$ $P_{H}=\left(\lambda+\frac{2}{3} \mu\right) \theta$, where $\theta(z)$ is the cubic dilation, and $\lambda(z)$ and $\mu(z)$ are Lamés parameters described before.

A very important physical feature is the pressure discontinuity across interfaces layers $\left(\Delta P=P^{+}-P^{-}\right)$, at the depth $z$ (positive down), which exists if the velocity ratio, $\gamma$, exhibits a discontinuity. Considering the case of a medium formed by planehorizontal layers, and Hooke's isotropic law, it is shown that the discontinuity $\Delta P$ is given by

$$
\Delta P(z)=\frac{4}{3}\left(\gamma_{1}^{2}-\gamma_{2}^{2}\right) P_{0}(z),
$$

where $\gamma_{1}$ is the upper layer and $\gamma_{2}$ the lower layer parameters across the interface positioned at depth $z$, with the overload pressure varying by positive and negative jumps.

This idea may seem a little strange in simple geological descriptions, but it is a fact related to the non-elementary behavior of stress in solids. The mentioned interfaces are goals in the geological interpretation of seismic sections, and examples are shown in Figure 1.

The intensity of tangential stress, $P_{T}$, is a way of measuring the mechanical instability responsible for the capacity to destroy a granular structure, and to produce fracture in a solid rock, and the following result is obtained:

$$
P_{T}(z)=\frac{1}{2}\left(\sigma_{z z}-\sigma_{x x}\right)=\gamma^{2} P_{z}(z),
$$

where again this result also depends on the $\gamma$ ratio.

\section{RESULTS}

The velocities distribution used were based on controlled empirical models with the parameters presented in the Table 1 and illustrated in Figure 2, where A1 represents the water layer (dark blue), A2 a formation overlying the reservoir (light blue), A3 the reservoir formation (yellow), A4 the formation that includes the source rock (reddish orange), and A5 the basement (brownish red).

Table 1 - Example of used parameters.

\begin{tabular}{|c|l|l|l|l|l|}
\hline Parameter & $\mathrm{A} 1$ & $\mathrm{~A} 2$ & $\mathrm{~A} 3$ & $\mathrm{~A} 4$ & $\mathrm{~A} 5$ \\
\hline$\gamma_{0}$ & 0 & 0.687 & 0.807 & 0.677 & 0.570 \\
$v_{P 0}$ & 1500 & 2280 & 3442 & 4222 & 5002 \\
$k_{P}$ & 0 & 1.5 & 0.5 & 0.75 & 0.5 \\
$k_{g}$ & 0.0001 & 0.0001 & 0.0001 & 0.0001 & 0.0001 \\
\hline
\end{tabular}

The formula for the velocity $v_{P}$ in each geological formation was defined by $v_{P}(z)=v_{P 0}+k_{P} z$, with a small gradient $k_{P}$. For the $\gamma$ distribution was also admitted a linear function, $\gamma(z)=\gamma_{0}+k_{g} z$, with also a small gradient $k_{g}$. For the density distribution, as $\rho\left(v_{P}\right)=0.452+0.4788 v_{P}$ adopted from Mavko et al. (1999). These models were applied to the interpreted seismic-stratigraphic sections of Figure 1; to emphasize, these formulas were applied along $z$ for each point of the $x$ axis; in this way, the maps show directly the matrix distributions for $v_{P}(x, z)$, $v_{S}(x, z), \rho(x, z)$, and consequently $\gamma(x, z)$. 


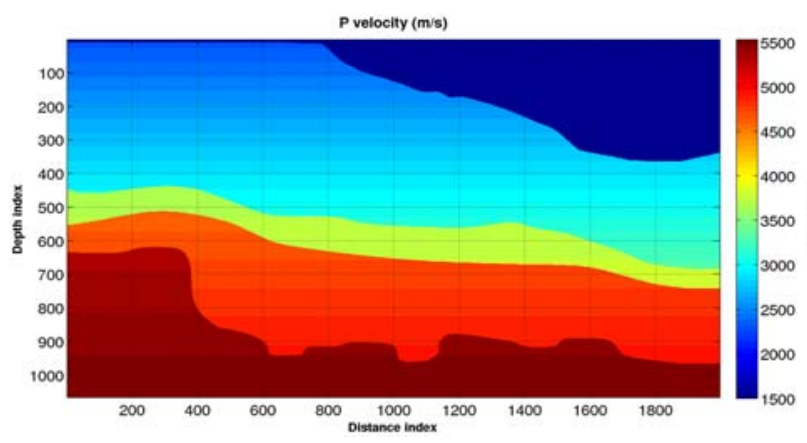

(a) Line 66 .

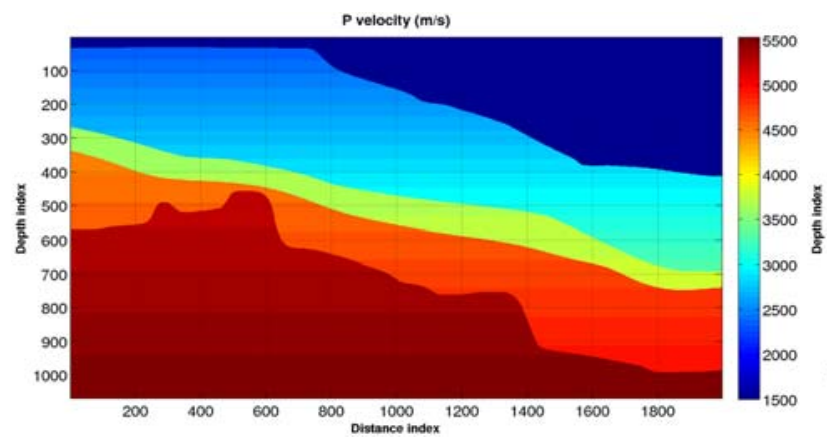

(c) Line 70

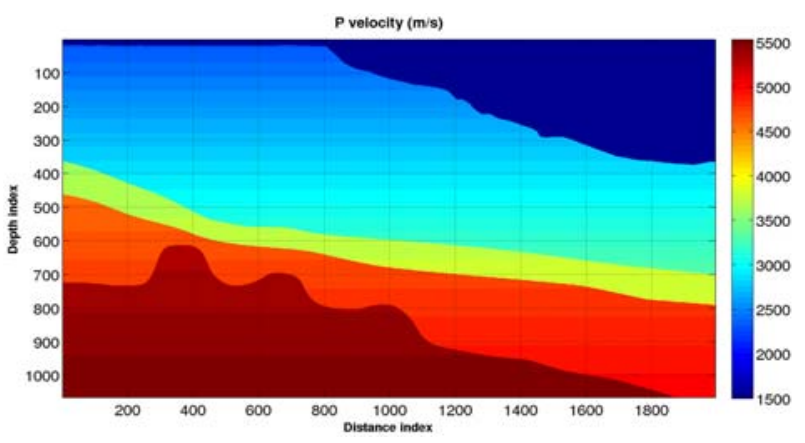

(b) Line 68.

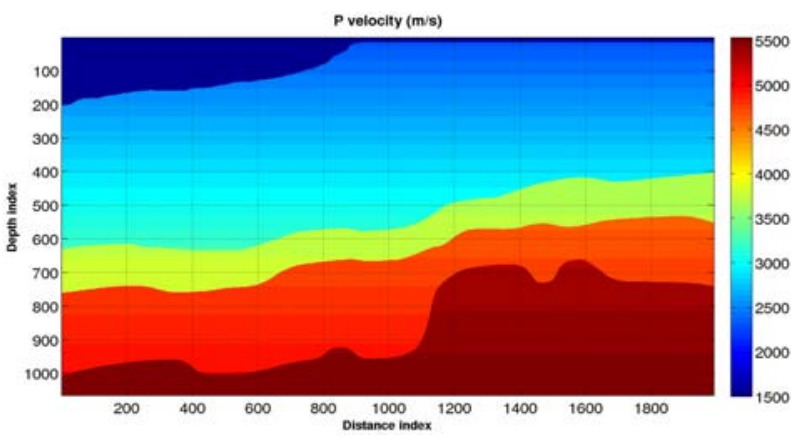

(d) Line 97 .

Figure 2 - Velocity, $v_{P}$.

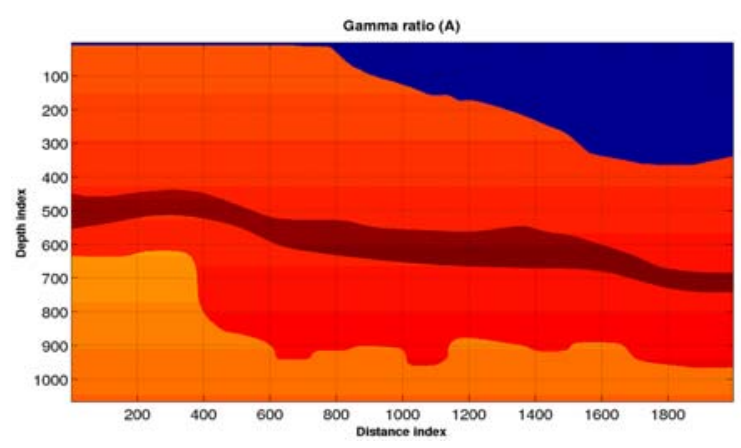

(a) Line 66

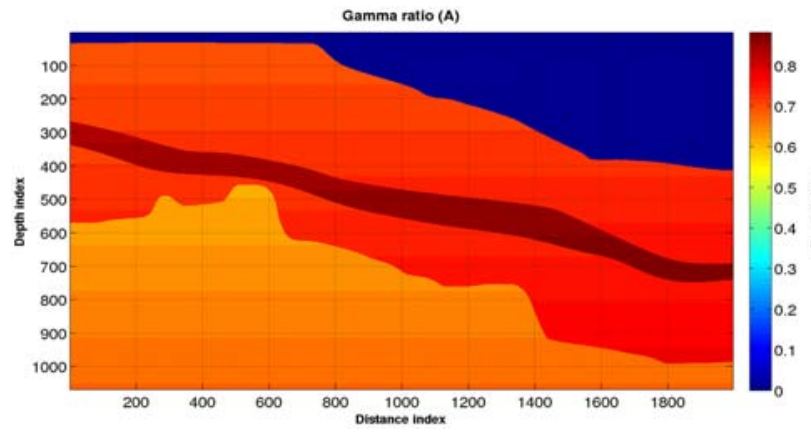

(c) Line 70

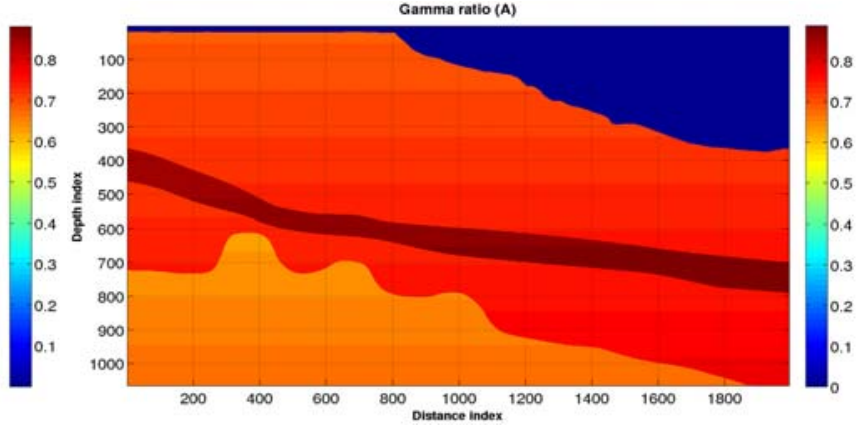

(b) Line 68

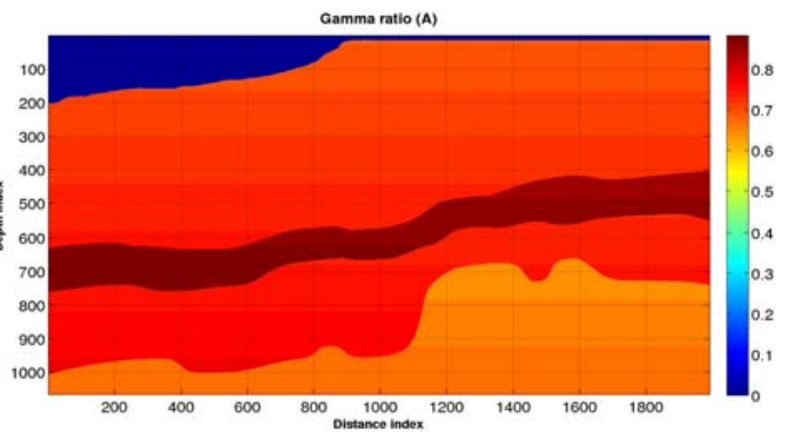

(d) Line 97.

Figure 3 - Gamma ratio, $\gamma$. 


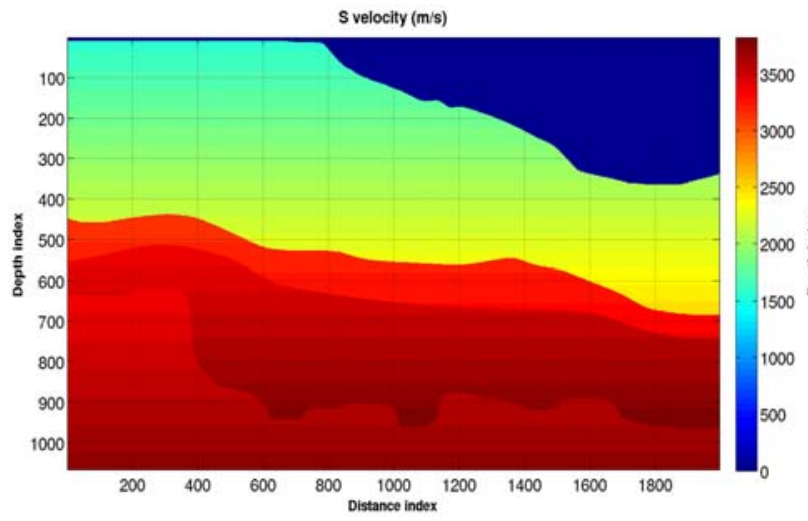

(a) Line 66 .

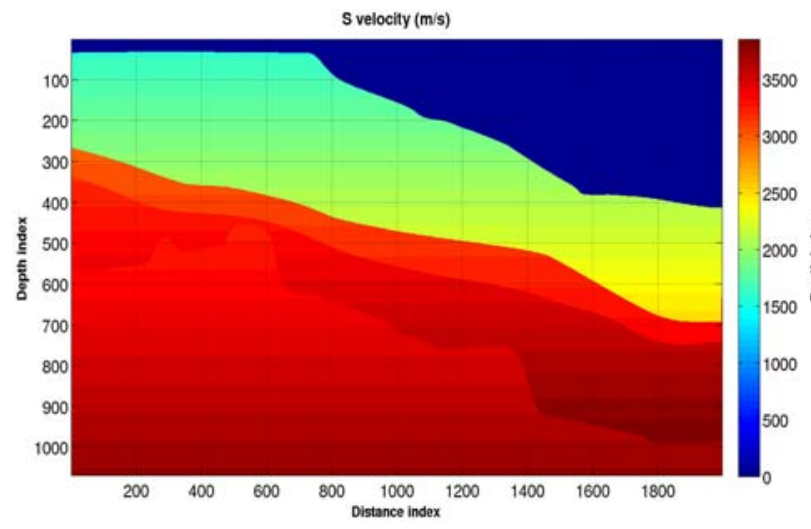

(c) Line 70

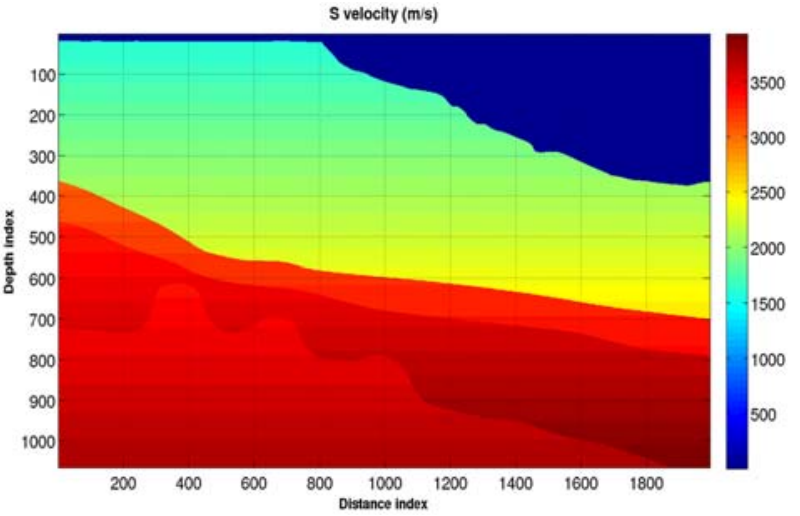

(b) Line 68 .

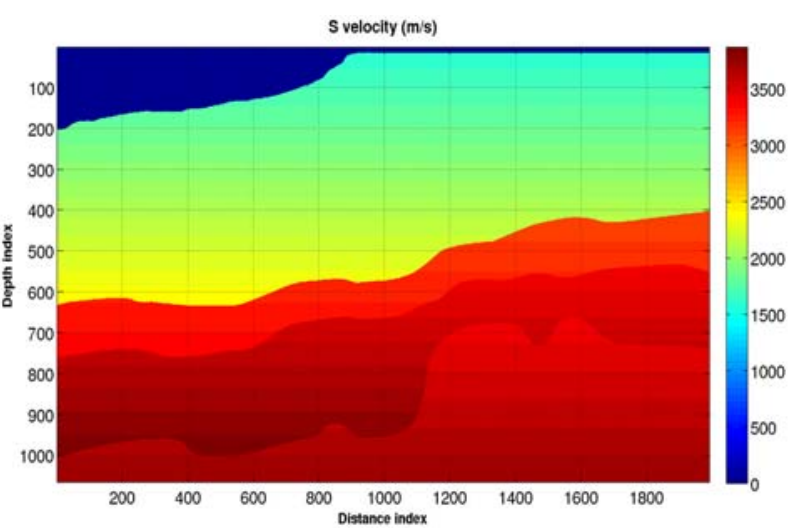

(d) Line 97.

Figure 4 - Velocity, $v_{S}$.

The basic sections to start the pressure prediction are the velocity $v_{P}$ and the $\gamma$ ratio, from where the other parameters were calculated (see Figs. 2, 3 and 4). In order to obtain these sections for the parameter distributions, we started from the CRS stack interpreted sections, where the source and reservoir formations were selected based on some geological information.

The empirical $v_{P}$ models are of mixed type; i.e., composed of jumps and smooth linear trend; thus, for the rock formations a vertical gradient was assigned to the $\mathrm{P}$ wave velocity in the discrete form $v_{P}(n)=v_{P 0}+k_{P} n$ (in the experiment $k$ varied between 0.1 and 0.7 ), where $\Delta z$ is included in the $k_{P}$ factor. This also means that we looked only at the data as a matrix, where can be applied a rather complex change of scale to transform from time $(t)$ to depth $(z)$, or simply by using a constant average velocity value. A similar formulation was applied to the $\gamma(z)$ distribution. From the $v_{P}(x, z)$ and $\gamma(x, z)$ a priori informations, the distribution for $v_{S}(x, z)$ was calculated and, therefore, for the other desired parameters.
Figure 5 shows the density distribution $\rho(x, z)$ for the example, which also followed the mixed formalism similar to that for $v_{P}$; that is, with jumps and smooth vertical gradients.

In the following figures for the different types of pressure calculation, we look for zones of low and high values. Figures for the vertical pressure, $P_{z}$, calculated by Eq. (7), showed a very smooth behavior as expected, and for the sake of space are not presented here. Figure 6 of the horizontal pressure, $P_{x}$, calculated by Eq. (8), shows a very distinct and special behavior in each section. Figure 7 of the deviatoric pressure between vertical and horizontal pressures, $P_{T}$, calculated by Eq. (11), shows a very special behavior for the low and high value zones, and either positive or negative, and the information is that the vertical pressure exceeds the horizontal pressure that can act as a reservoir zone.

The hydrostatic pressure (solid phase) of Figure $8, P_{H}$, calculated by Eq. (9), clearly models the low pressure zone (central blue stripe) between two high pressure zones (weaker above and stronger below). But it is interesting that part of the basement presents zones of intermediate pressure. 


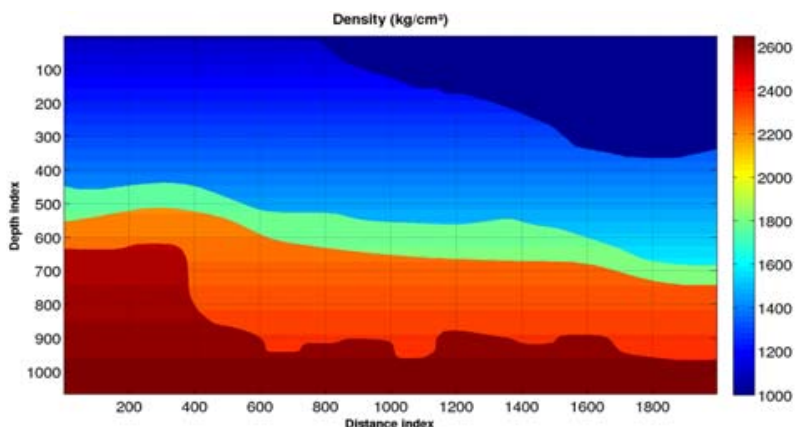

(a) Line 66

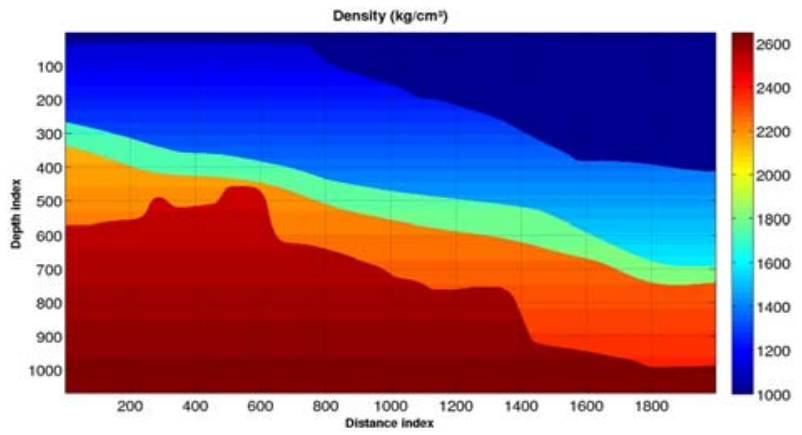

(c) Line 70

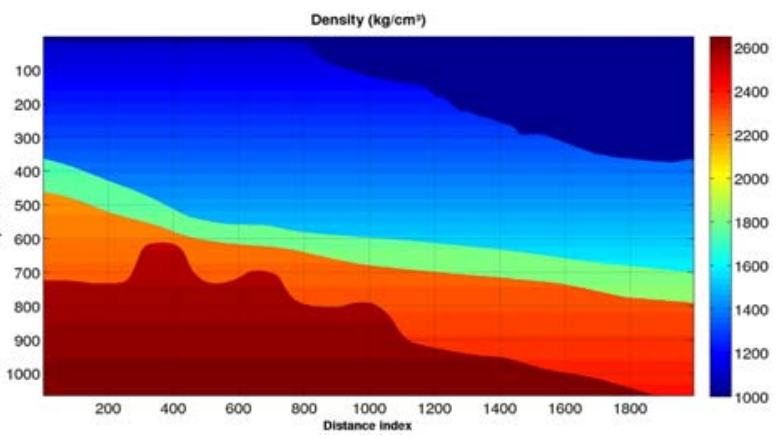

(b) Line 68

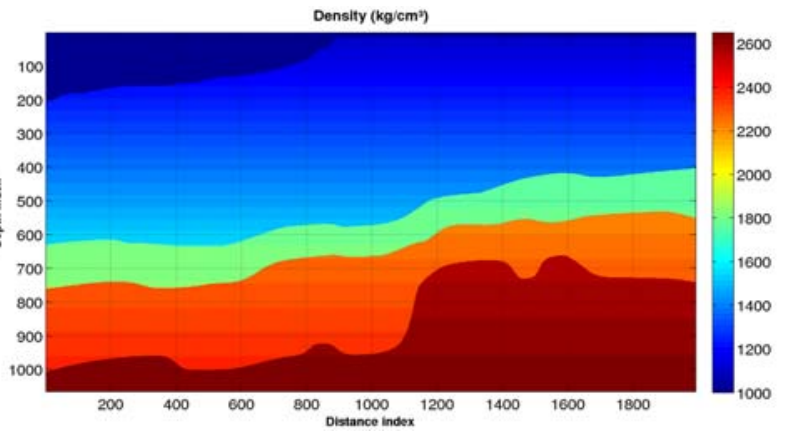

(d) Line 97.

Figure $\mathbf{5}$ - Density, $\rho$

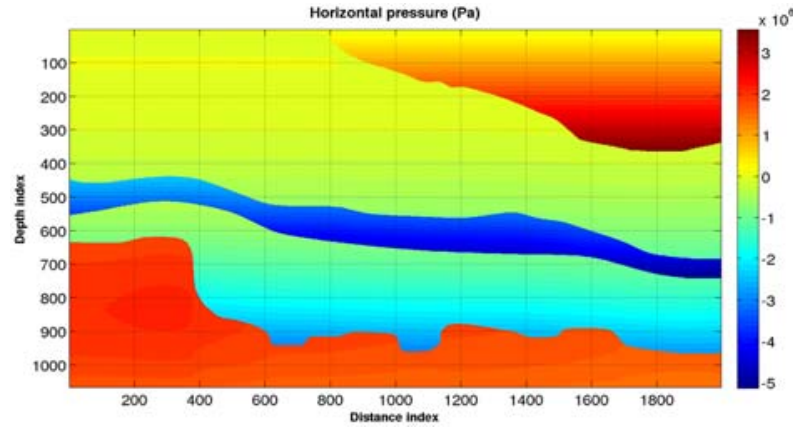

(a) Line 66

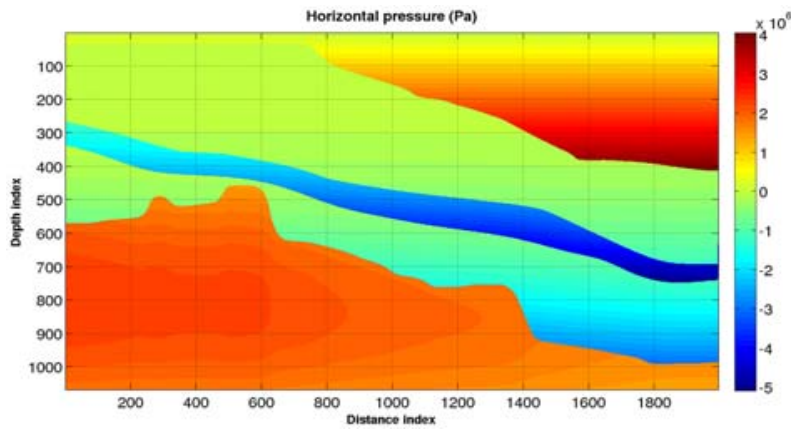

(c) Line 70

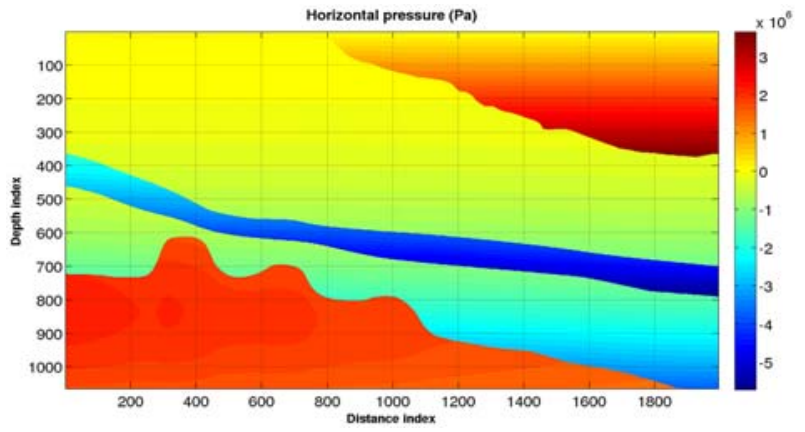

(b) Line 68.

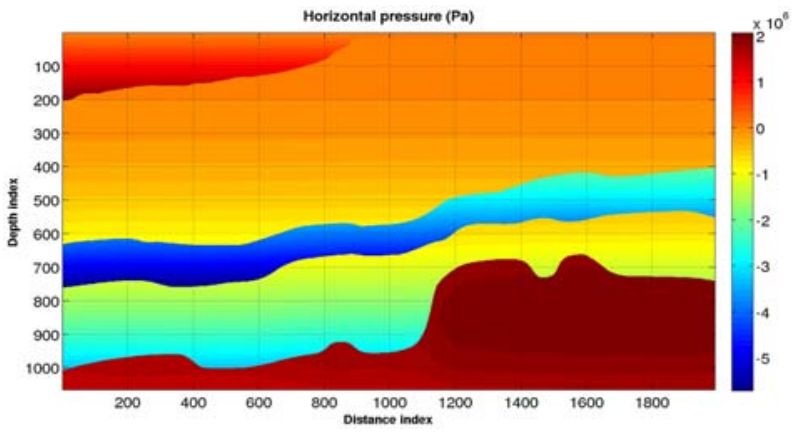

(d) Line 97.

Figure 6 - Horizontal pressure, $P_{x}$. 


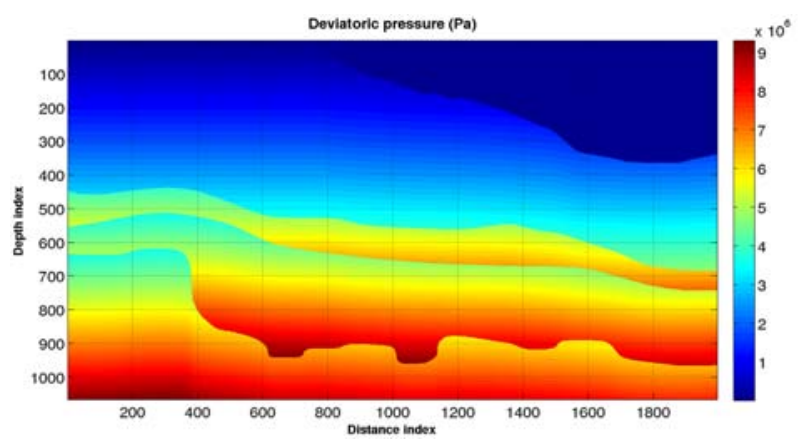

(a) Line 66.

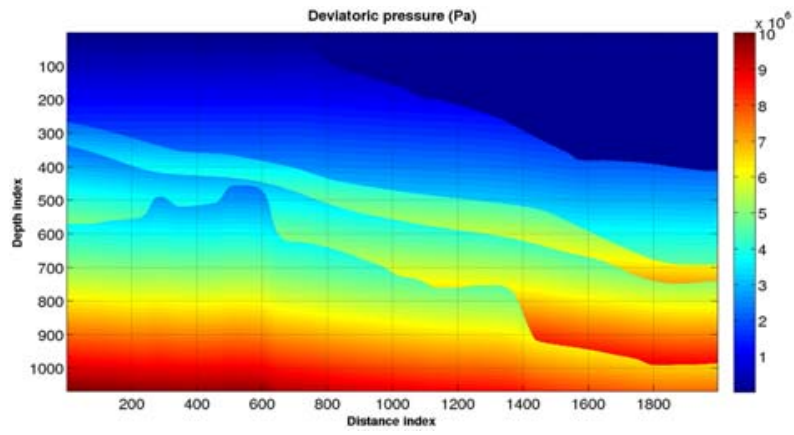

(c) Line 70

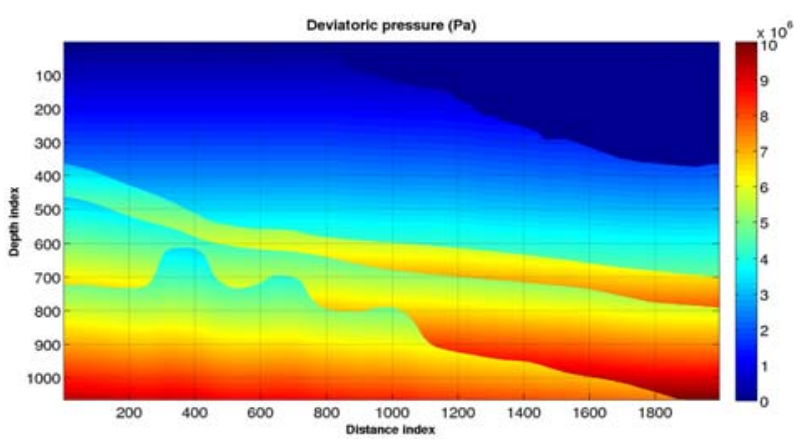

(b) Line 68.

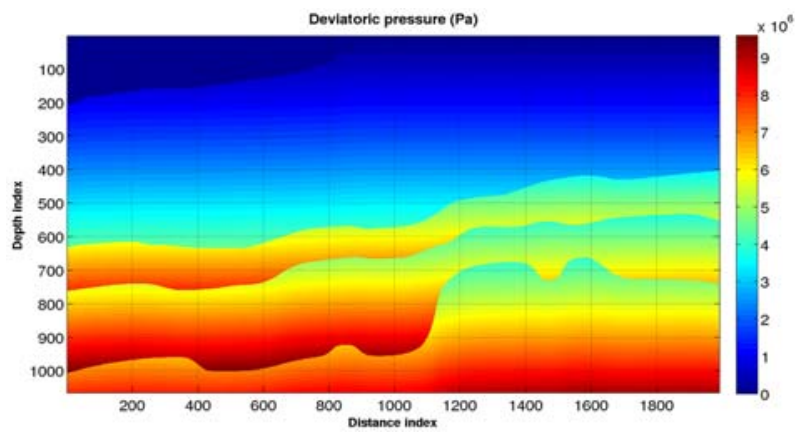

(d) Line 97.

Figure 7 - Deviatoric pressure, $P_{T}$.

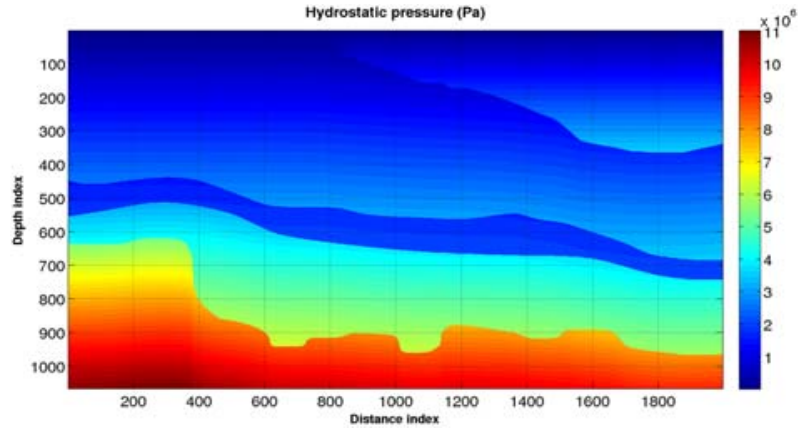

(a) Line 66.

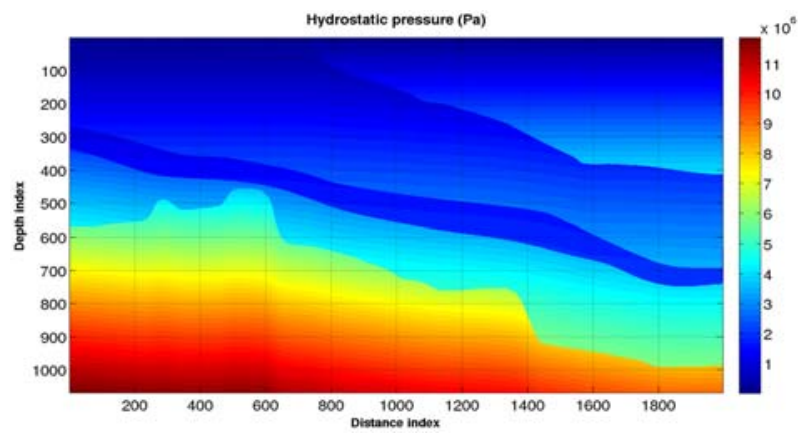

(c) Line 70 .

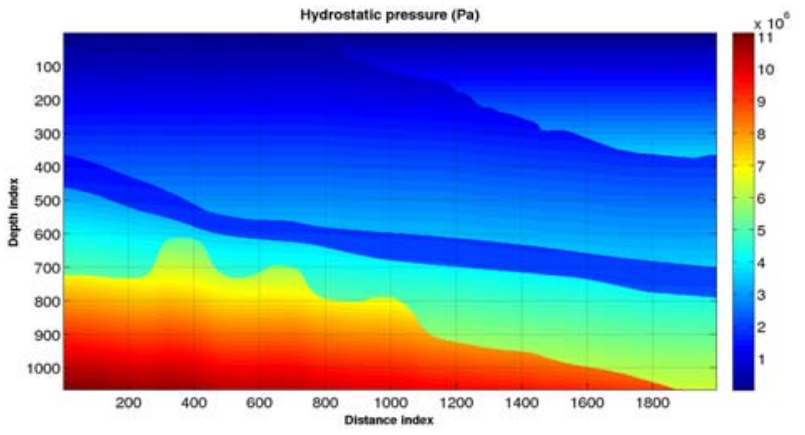

(b) Line 68 .

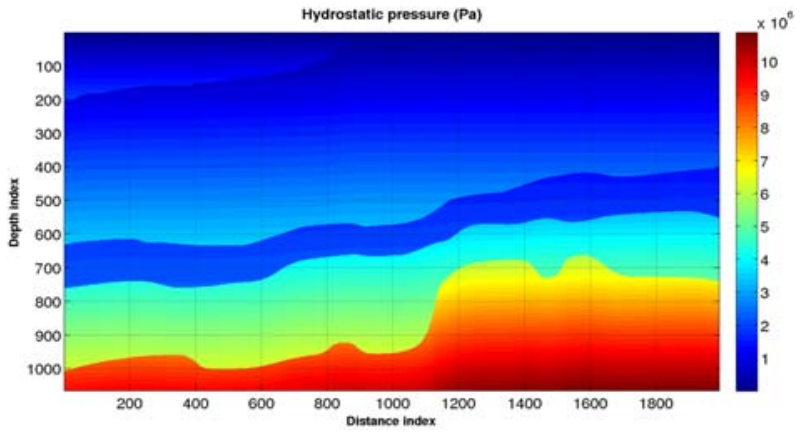

(d) Line 97 .

Figure 8 - Hydrostatic pressure, $P_{H}$. 
Figures of the pressure jump,

$$
\Delta P(z)=\frac{4}{3}\left(\gamma_{1}^{2}-\gamma_{2}^{2}\right) P_{0}(z)
$$

( $\gamma_{1}$ is above and $\gamma_{2}$ below the separating interface), expose faint lines of the discontinuities that serve to mark the different formations, and the quality of the process, but they were not presented here due to to space. Similarly, figures of the Poisson's coefficient, $\nu=\frac{\Delta x}{\Delta z}$, clearly model, in some detail, negative values for the low pressure zone, and smooth gradients for the other formations, and positive values.

Figures 9, 11 and 13 represent the called empirical cubes for the contents of horizontal, hydrostatic and deviatoric pressures, respectively. These cubes were constructed by simple linear interpolation, after placing the three parallel sections (lines 66, 68, 70 ) in their respective positions as an empirical cube controlled by a discrete, $(i, j, k)$ grid, with established spacings $\Delta x=383 \mathrm{~m}$ and $\Delta y=195 \mathrm{~m}$. The value for $\Delta z$ will depend on the scale of the transformation of the time axis, $t$, to depth, $\Delta z$; for example, admitting a total depth of $8000 \mathrm{~m}$ for the total number of points (200), then we have approximately $\Delta z=40 \mathrm{~m}$. Also the vertical scale can be constructed using an average velocity, or a depth migration operator. A reservoir formation usually has a rather small thickness (for instance, $5 \mathrm{~m}, 10 \mathrm{~m}, 20 \mathrm{~m}$ ); therefore, to follow a reservoir laterally one needs to make horizontal sections in the 3D cube; but the main idea here is to look at the lateral extensions of the target zone (formation mapped as the reservoir), since it can have a rather large dip across the section. The matrix representing the cube is $200 \times 107 \times 41$ points. The correspondent geological cube is $x=42 \mathrm{~km}, y=12 \mathrm{~km}$, and $z=7 \mathrm{~s}$ (1750 points in the time scale, with a discretization of $4 \mathrm{~ms}$ ). These values were established due to the available computer power; the finer the sampling, better details can be seen.

Following this main idea, Figures 10, 12 and 14 represent horizontal sections of the respective cube, that were selected based on the vertical index (also called depth index), therefore they represent maps of the variation of pressure for different levels. Looking at these horizontal sections we can notice the nonuniform sampling in the $(x, y, z)$ directions, resulting in square edges, while the vertical sections (Figs. 9, 11 and 13) are smooth. We make clear and inform that only a linear interpolation was performed to construct the cubes, although other interpolation options exist like cubic spline.

The main idea is to combine pairs, Figures 9 and 10, 11 and 12,13 and 14, in order, first, to interpret the subsurface continuation of a productive reservoir, and second to examine potential zones as reservoirs. The present analysis is very specific, and takes in consideration the mapping of zones of low and high pressures. Looking at the combined pairs of pressure distributions (horizontal, hydrostatic and deviatoric), we should follow the evolution of the blue (bluish) color of the target zone horizontally and vertically.

With these considerations, we call attention to the target horizontal pressure with the form of a blue strip crossing the vertical sections from left to right, with a gentle dip, and can be clearly seen in Figure 9, for an easy description. Now following Figure 10, the target presents a lateral extension, and a sharp confined zone at the level depth index 60 . This effect can also be seen in the Figure 12 for the hydrostatic pressure, but for the deviatoric pressure (Fig. 14) the color pattern changes to indicate the concept for variation between hydrostatic and horizontal pressures.

\section{CONCLUSIONS}

Pressure prediction is a very attractive subject, but not so simple and deserves a lot of attention to the physical-mathematical modeling as a boundary value problem.

The seismic data were processed with CRS technology proved to be more effective in the geometrical delineation of reflectors of the stacked and migrated sections, that were used to construct an empirical velocity $\left(v_{P}\right.$ and $\left.v_{S}\right)$ and density $(\rho)$ models based on the concept of an existing productive reservoir, where the $\gamma$ distribution is the constraint to indicate its presence.

The mapping of low pressure zones, corresponding to a proposed reservoir formation, has a spatial extension that depends on the seismic-stratigraphic and structural geology, consequently on their interpretation.

This research topic is attractive in the sense that it is a datadriven method aiming to extend, or discover, a low pressure zone for a successful drilling.

A more complete continuation of this work would require more seismic data and log information. 3D surveys would be ideal, besides borehole, structural, and tectonic parameters for the area; but, this type of information has limits to public use for academic studies due to property contracts.

\section{ACKNOWLEDGEMENTS}

The authors would like first to thank the reviewers for their patience and positive contributions. To thanks are extended to the Brazilian institution UFPA (Universidade Federal do Pará), and to the project National Institute of Science and Technology (Instituto Nacional de Ciência e Tecnologia, INCT-GP, of MCT/CNPq/FINEP) for the research support aiming at oil and gas exploration. 


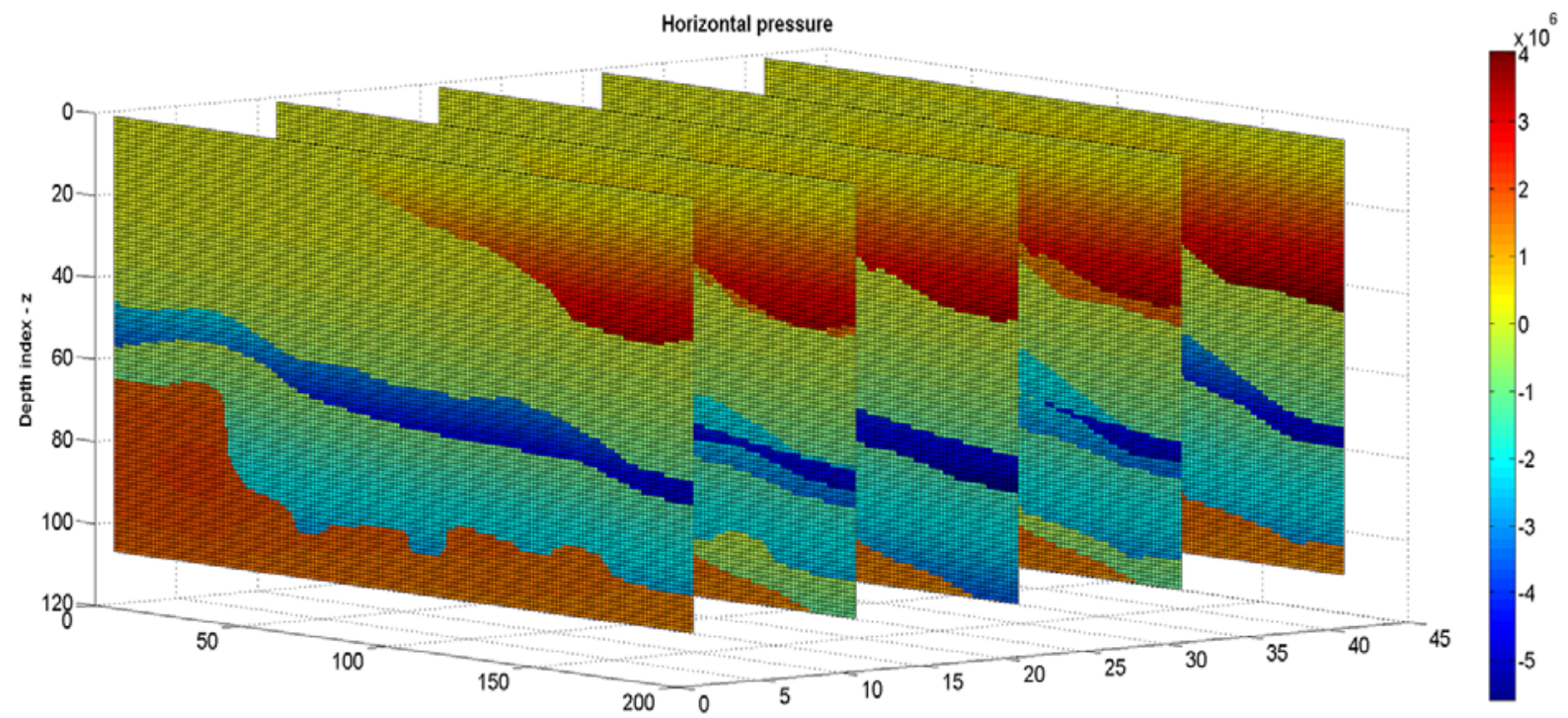

Distance index $\cdot x$

Distance index $-y$

Figure 9 - Vertical sections of the empirical cube for horizontal pressure $P_{x}(x, y, z)$.

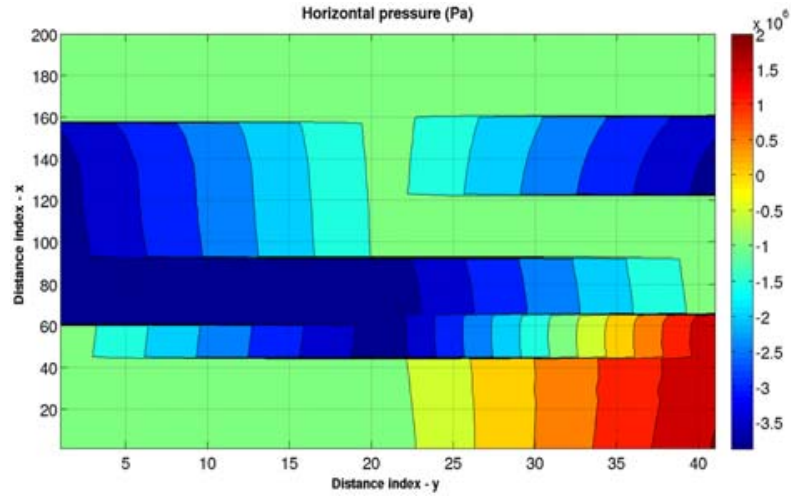

(a) Depth index $=60$.

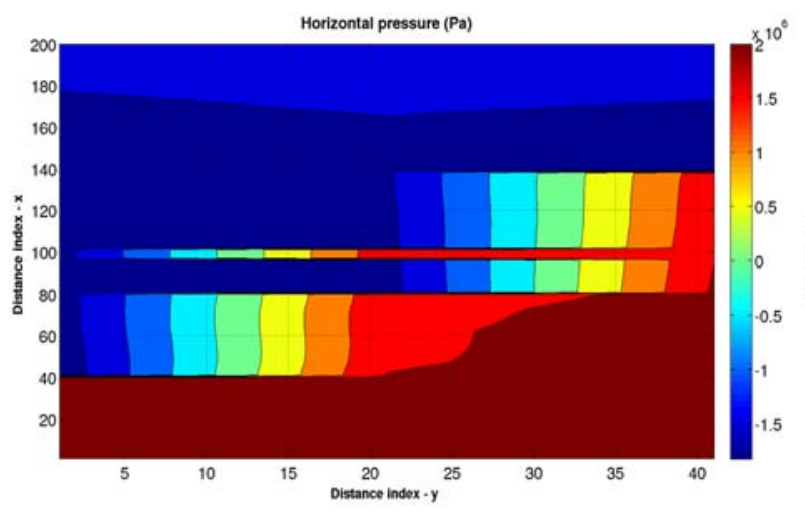

(c) Depth index $=80$.

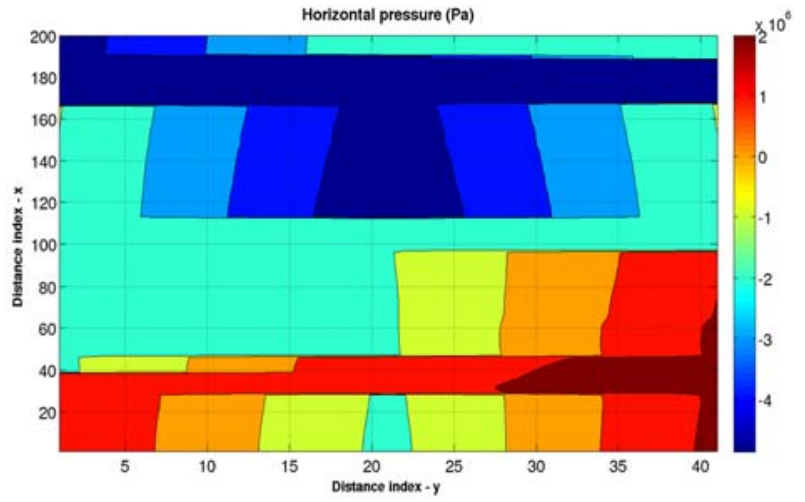

(b) Depth index $=70$.

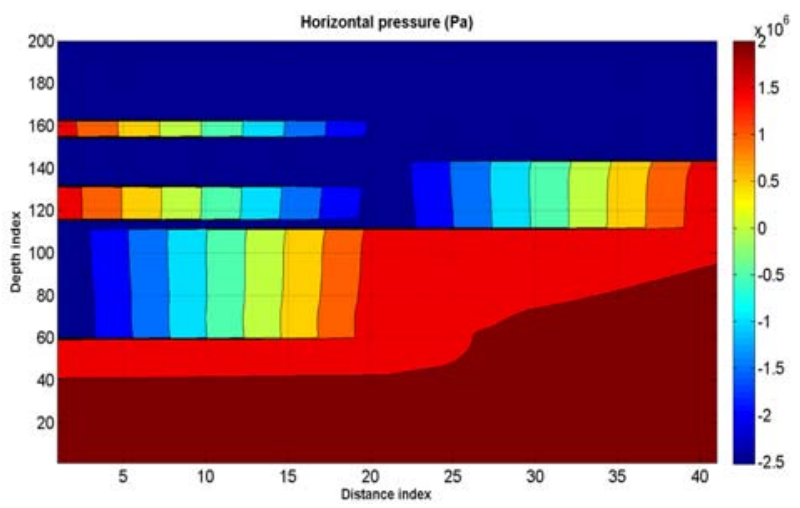

(d) Depth index $=90$.

Figure $\mathbf{1 0}$ - Horizontal sections of the empirical cube for horizontal pressure $P_{x}(x, y, z)$. 


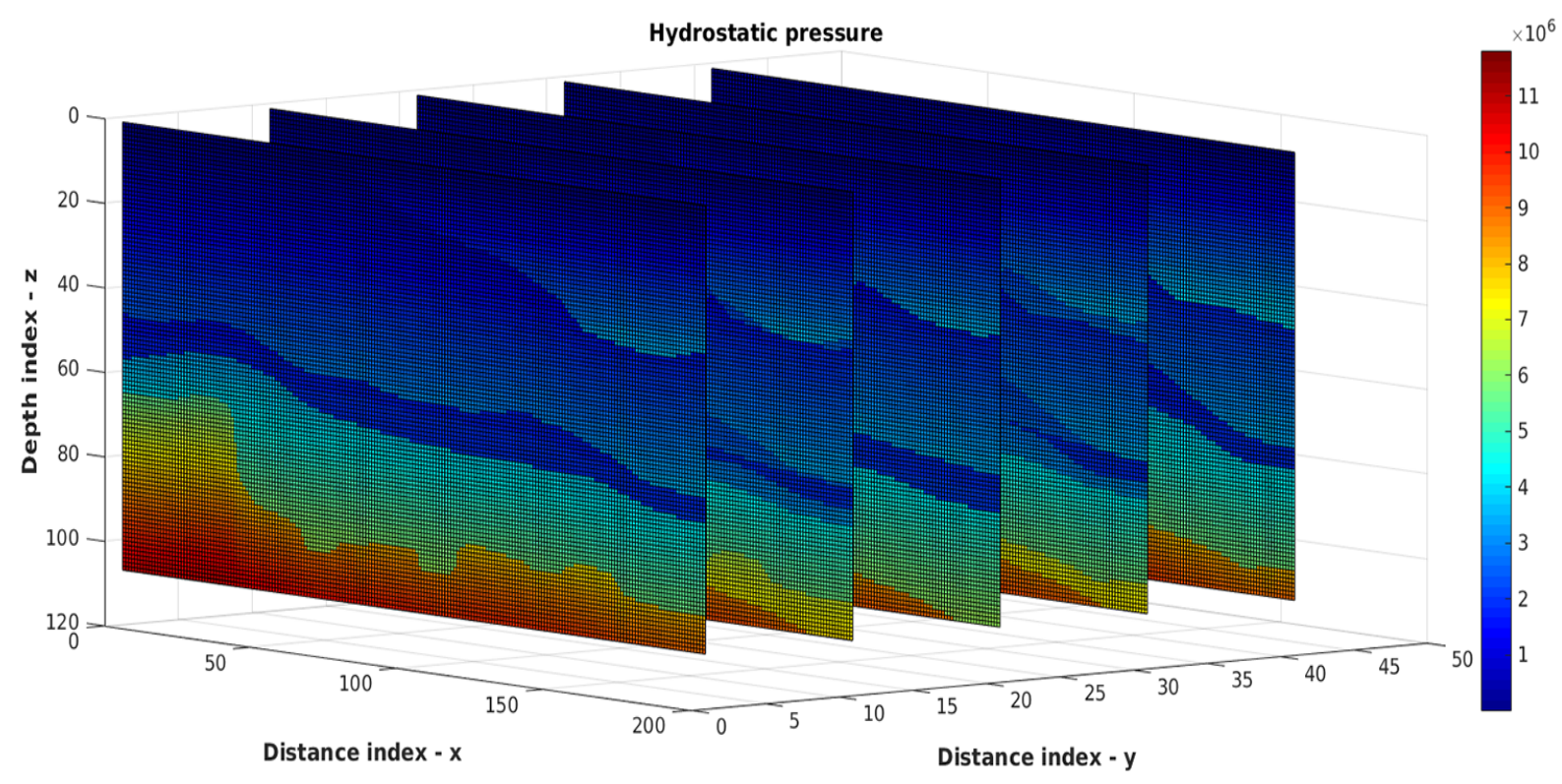

Figure 11 - Vertical sections of the empirical cube for hydrostatic pressure $P_{H}(x, y, z)$.

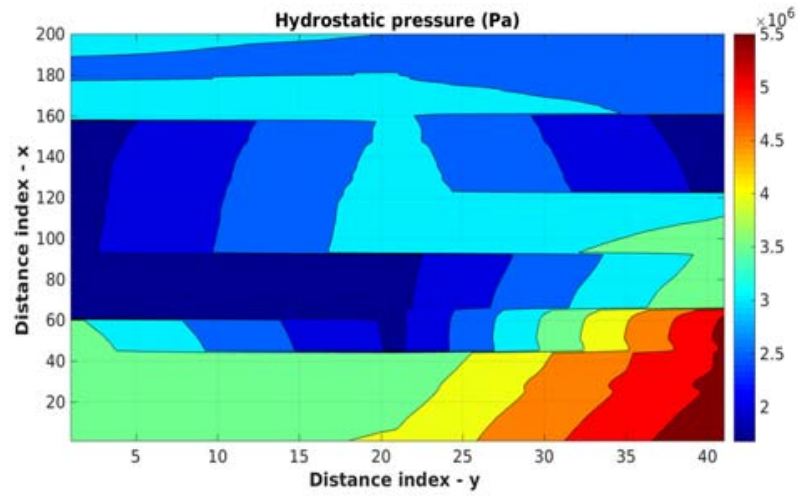

(a) Depth index $=60$.

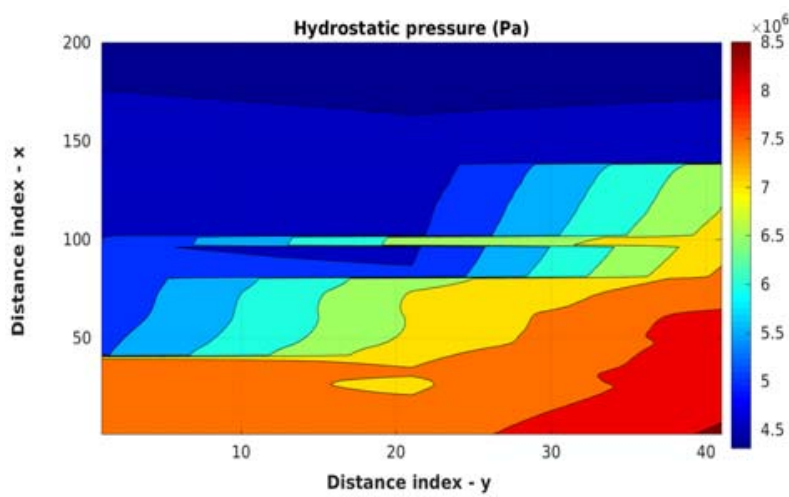

(c) Depth index $=80$

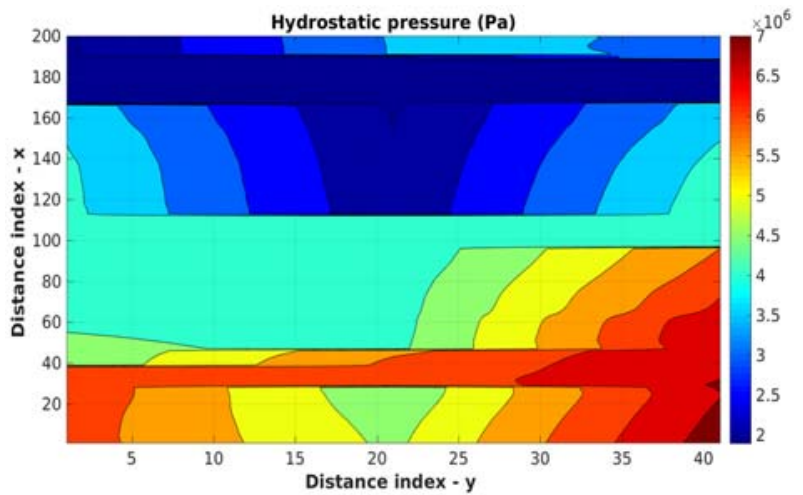

(b) Depth index $=70$.

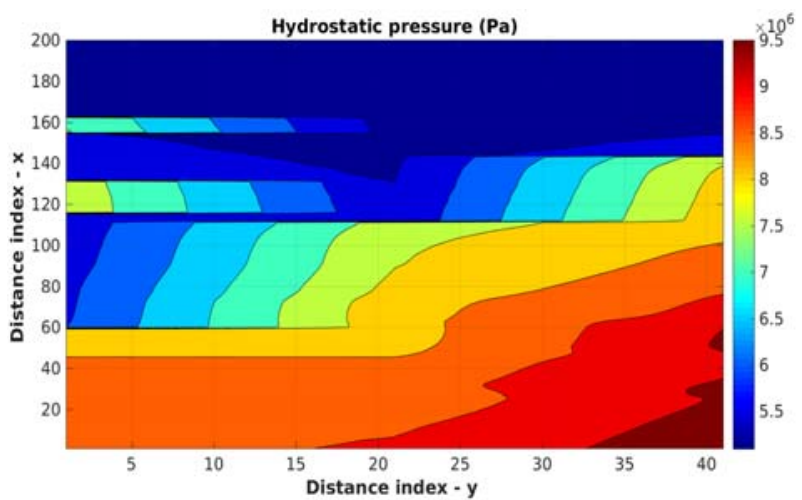

(d) Depth index $=90$.

Figure 12 - Horizontal sections of the empirical cube for hydrostatic pressure $P_{H}(x, y, z)$. 


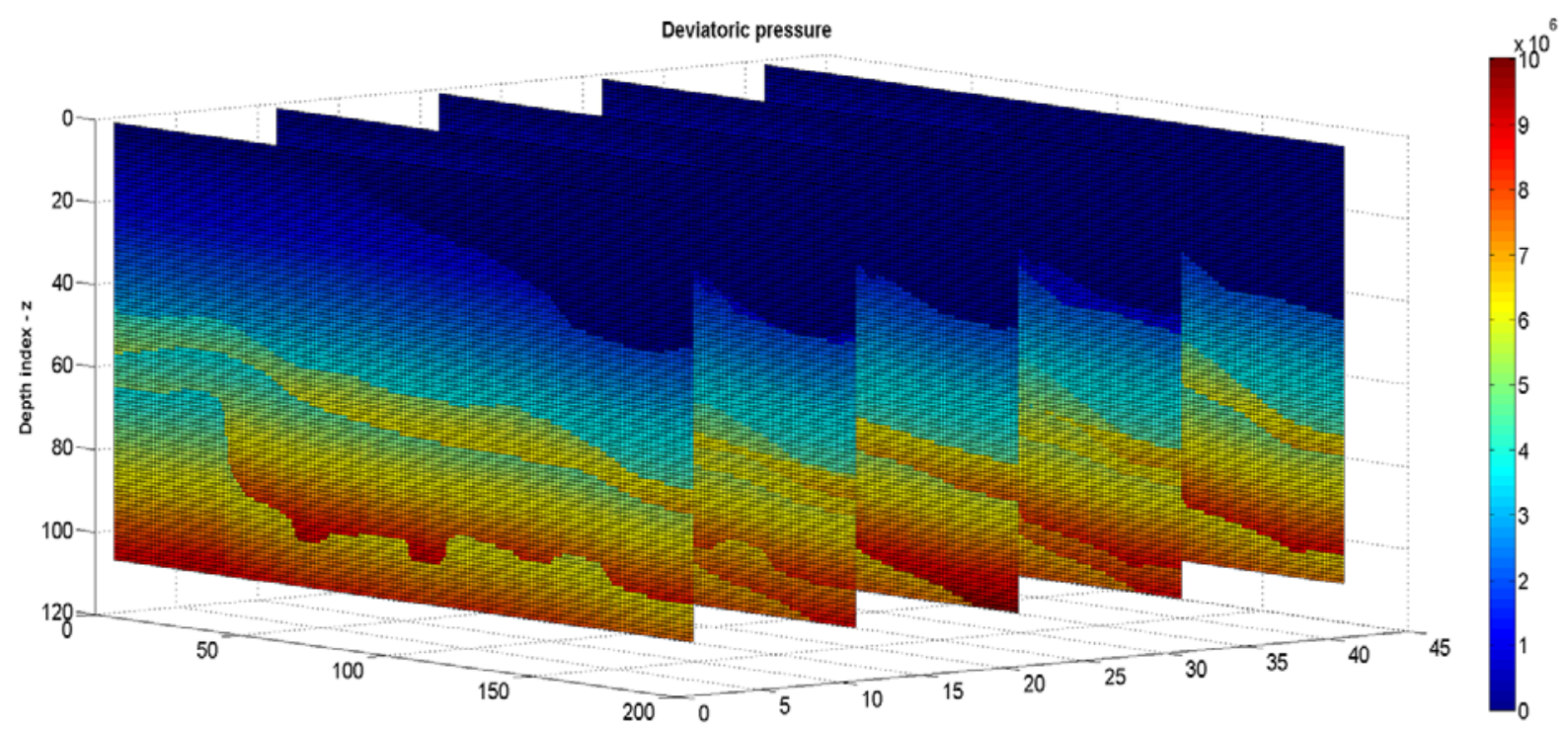

Distance index $\cdot x$

Distance index - $y$

Figure 13 - Vertical sections of the empirical cube for deviatoric pressure $P_{T}(x, y, z)$.

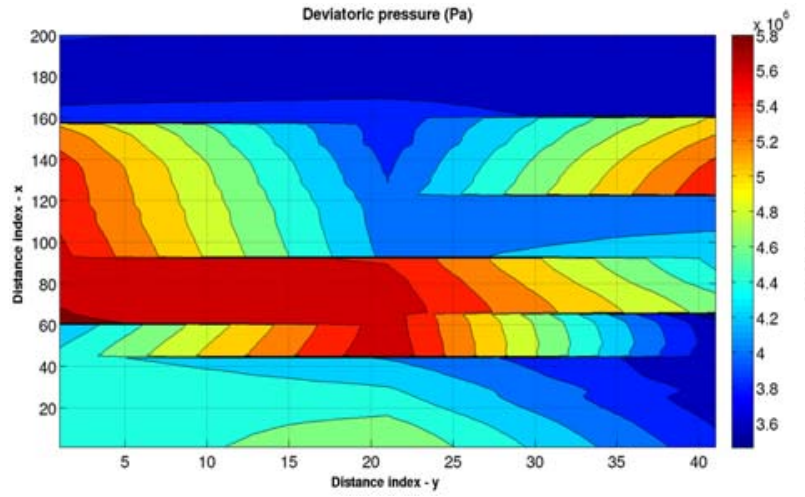

(a) Depth index $=60$.

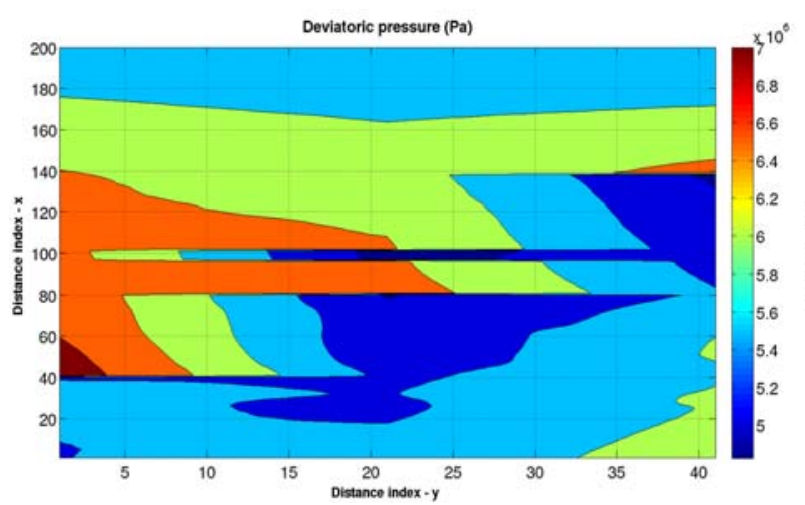

(c) Depth index $=80$.

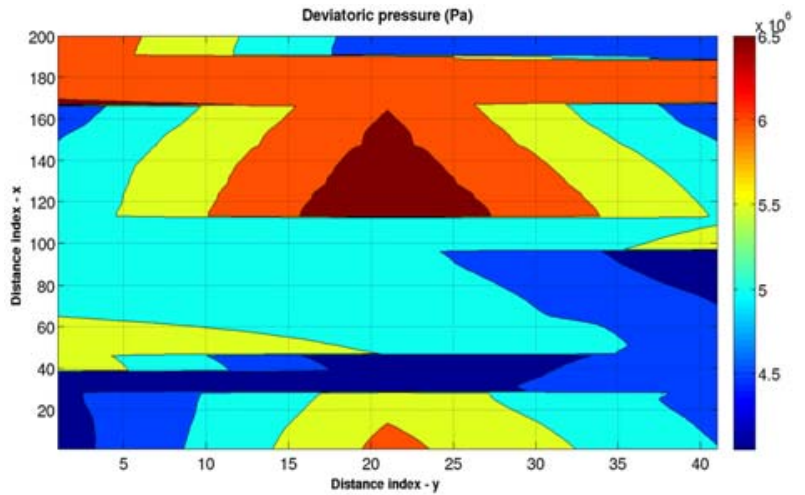

(b) Depth index=70.

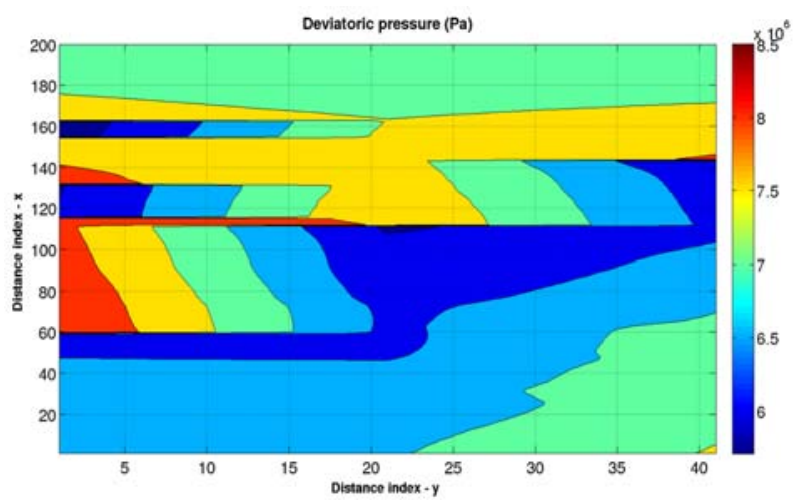

(d) Depth index $=90$.

Figure 14 - Horizontal sections of the empirical cube for deviatoric pressure $P_{T}(x, y, z)$. 


\section{REFERENCES}

AMEEM MS. 2003. Fracture and in-situ stress characterization of hydrocarbon reservoirs. London: The Geological Society of London. 216 pp.

HANTSCHEL T \& KAUERAUF Al. 2009. Fundamentals of basin and petroleum systems modeling. Berlin: Springer-Verlag. 476 pp.

LEITE LWB, VIEIRA WWS \& SIBIRYAKOV BP. 2016. Subsurface stresS prediction using seismic data for oil and gas exploration. Brazilian Journal of Geophysics, 34(1): 95-116.

MANN J. 2002. Extensions and Applications of the Common-Reflection-Surface Stack Method. Doctorade Thesis - Karlsruhe University, Karlsruhe. 165 pp.

MAVKO G, MUKERJI T \& DVORKIN J. 1999. The rock physics handbook. London: Cambridge University Press. 339 pp.

MELLO MR, GONÇALVES FTT \& NETTO AST. 1994. A successful application of the petroleum system concept in the Camamu basin offshore
Brasil. In: First Point AAPG/AMPG Research Conference Geological Aspects of Petroleum Systems, Mexico City, Mexico.

PAYTON CE. 1977. Seismic stratigraphy - applications to hydrocarbon exploration. Tulsa, OK: American Association of Petroleum Geologists. (Memoir, 26). $516 \mathrm{pp}$.

PERSEN LN. 1975. Rock dynamics and geophysical exploration. Amsterdam, Holland: Elsevier Scientific Publishing Company. 270 pp.

SIBIRYAKOV EP, LEITE LWB \& VIEIRA WWS. 2014. Model of the structured continuum, and the relation between specific surface area, porosity and permeability. Brazilian Journal of Geophysics, 31(4): 559-568.

SIBIRYAKOV EP, LEITE LWB \& VIEIRA WWS. 2015. Behavior of stresses in structures and the effect on hydrodynamics analyzed from multicomponent seismic data. Brazilian Journal of Geophysics, 33(1): 57-70.

SILVA ANP. 2016. Processamento, imageamento e cálculo de predição de pressão em dados sísmicos numa bacia sedimentar. Master Dissertation - UFPA, Belém, Pará, Brazil. 187 pp. 